\title{
ultramicroscopy
}

ELSEVIER

\section{Multiple scattering in low-energy electron holography}

\author{
Mark R.A. Shegelski*, Matthew Reid, Lara Pow \\ Department of Physics, University of Northern British Columbia, 3333 University Way, Prince George, British Columbia, \\ Canada V2N $4 Z 9$
}

Received 28 August 1999; received in revised form 9 February 2000

\begin{abstract}
The theory of the low-energy electron point source (LEEPS) microscope is reformulated in matrix form to readily account for multiple scattering. An algorithm is developed for the storage of the structure matrix and an iterative method is used to solve the matrix equation for the structure factor. Examples of small and large clusters of atoms are given to compare single and multiple scattering. A Kirchhoff-Helmholtz transform is used for the reconstruction. We find that in some cases the multiple scattering is too strong and reconstruction is not possible. We give examples which show that, even when multiple scattering is important, one can still obtain reconstructions that reveal the atomic structure both along and lateral to the optical axis. We also compare our results with those found in LEED. (C) 2000 Elsevier Science B.V. All rights reserved.
\end{abstract}

Keywords: Electron holography; Reconstruction; Multiple scattering

\section{Introduction}

More than 50 years ago, D. Gabor [1] proposed a new principle of microscopy to overcome the limitations of lenses. Using a coherent ensemble of particles with wave nature, the fraction of the beam elastically scattered by an object is made to interfere with a coherent reference wave at some two-dimensional detector creating a hologram that contains information on both the amplitude and the phase of the scattered wave. The laser provides such a coherent source of photons for light holography. More recently, electron holography has been demonstrated in electron microscopy

\footnotetext{
*Corresponding author. Tel.: +1-250-960-6663; fax: + 1250-960-5545.

E-mail address: mras@unbc.ca (M.R.A. Shegelski).
}

[2,3], in photoemission electron holography [4], and in lensless low-energy electron microscopy [5-9]. Reviews of electron holography have been collected by Tonomura [10] (see in particular the article by Fink et al). The breakthrough in lowenergy holography was made by Fink $[5,6]$, who created an ultrathin metal tip with one or a few atoms at its apex to serve as a "point" source for a coherent electron beam [6-8], with a virtual source size of atomic dimensions as determined from interferometric measurements [11] and capable of emitting currents up to milli-Amperes [12]. In the low-energy electron point source (LEEPS) microscope the electrons are accelerated to energies in the range of $20-200 \mathrm{eV}$ and scatter off the atoms of a sample a distance $d=0.1-1 \mu \mathrm{m}$ away. On a screen a distance $D \approx 10 \mathrm{~cm}$ away an image is formed by the unscattered and the 
scattered electrons with a magnification of $D / d$. If the object only blocks a small fraction of the incoming electron beam, most electrons arrive on the screen unscattered and we have the typical situation of an in-line hologram.

A scattering theory has been developed to simulate LEEPS images, and an algorithm has been devised for the reconstruction of the wavefront at the object [13]. The theoretical development has so far been restricted mainly to single scattering. We have therefore set ourselves the task in this paper to explore the implications of multiple scattering, in particular to discern the quality of the reconstructed images. To this end we will elaborate a matrix method [14] to deal with multiple scattering in clusters. For a cluster of $N_{\mathrm{s}}$ atoms scattering in all partial waves up to $L$ the square matrix involved is of dimension $N_{\mathrm{s}}(L+1)^{2}$, i.e. much too large to store for any but the smallest clusters. We have developed an algorithm that allows the construction of the matrix for a crystalline cluster from the knowledge of its distinct elements only. Examples will be given to assess the importance of multiple scattering and in particular its effect on the process of reconstruction. We will show that, even in situations where multiple scattering is important, our reconstruction algorithm allows a faithful reconstruction of the wavefront at the object with atomic resolution.

\section{Method}

The theory of electron holography uses a scattering approach based on the LippmannSchwinger equation [13]. One models the electron source as emitting a wave of spherical symmetry. The scattering wave function for a spherical incoming wave can then be written as

$$
\psi_{\text {out }}(\boldsymbol{r})=r^{-1} \exp (\mathrm{i} k r)[1+\tilde{\psi}(\boldsymbol{r})] \text {, }
$$

where

$$
\begin{aligned}
& \tilde{\psi}(\boldsymbol{r})=\sum_{i=1}^{N_{\mathrm{s}}} \exp \left(-\mathrm{i} k \boldsymbol{r}_{i} \cdot \boldsymbol{r} / r\right) \sum_{l m} \tau_{l} Y_{l m}(\hat{\boldsymbol{r}}) F_{l m}\left(\boldsymbol{r}_{i}\right), \\
& \tau_{l}=k^{-1} \sin \delta_{l} \exp \left(\mathrm{i} \delta_{l}\right),
\end{aligned}
$$

where the $Y_{l m}$ are spherical harmonics, $\boldsymbol{r}_{i}$ locates the ith atom, $k=2 \pi / \lambda=\sqrt{2 m E} / \hbar$ is the wave number of the electrons of wavelength $\lambda$ and energy $E$, and $\delta_{l}(E)$ is the phase shift for the scattering of an electron with angular momentum $l$ and energy $E$ off an atom in the solid. (The phase shifts can be obtained from LEED theory. ${ }^{1}$ )

Multiple scattering enters the scattering wave function (2) via the structure factor. The equation for the structure factor can be written as an inhomogeneous matrix equation. Let $\mathbf{F}$ denote a column vector whose transpose is given by

$$
\begin{aligned}
\mathbf{F}^{\mathrm{T}}= & {\left[F_{0,0}\left(\boldsymbol{r}_{1}\right), F_{1,-1}\left(\boldsymbol{r}_{1}\right), F_{1,0}\left(\boldsymbol{r}_{1}\right), F_{1,1}\left(\boldsymbol{r}_{1}\right),\right.} \\
& \left.\ldots, F_{L, L}\left(\boldsymbol{r}_{1}\right), \ldots, F_{L, L}\left(\boldsymbol{r}_{N_{s}}\right)\right],
\end{aligned}
$$

consisting of all structure factors for all relevant partial waves (up to $L$ ) at all $N_{\mathrm{s}}$ atomic positions. The matrix equation for $\mathbf{F}$ is

$$
\mathbf{F}=\boldsymbol{\Phi}+\mathbf{M F}
$$

or

$$
\mathbf{F}=\boldsymbol{\Phi}+\mathbf{M} \Phi+\mathbf{M}^{2} \boldsymbol{\Phi}+\mathbf{M}^{3} \boldsymbol{\Phi}+\ldots,
$$

where $\boldsymbol{\Phi}$ is a column vector with components

$$
\Phi_{l m}\left(\boldsymbol{r}_{i}\right)=4 \pi Y_{l m}^{(*)}\left(\hat{\boldsymbol{r}}_{i}\right) r_{i}^{-1} \exp \left(\mathrm{i} k r_{i}\right)
$$

and $\mathbf{M}$ is a matrix with zeros along its diagonal and with off-diagonal components given by

$$
\begin{aligned}
& M\left(\boldsymbol{r}_{i}, l, m ; \boldsymbol{r}_{j}, l^{\prime}, m^{\prime}\right) \\
& \quad=4 \pi Y_{l m}^{(*)}\left(\hat{\boldsymbol{r}}_{i j}\right) \tau_{l^{\prime}} Y_{l^{\prime} m^{\prime}}\left(\hat{\boldsymbol{r}}_{i j}\right) r_{i j}^{-1} \exp \left(i k r_{i j}\right), \\
& \boldsymbol{r}_{i j} \equiv \boldsymbol{r}_{i}-\boldsymbol{r}_{j}, \text { and } \hat{\boldsymbol{r}}_{i j} \equiv \boldsymbol{r}_{i j} /\left|\boldsymbol{r}_{i j}\right| .
\end{aligned}
$$

The solution to the multiple scattering problem is thus reduced to a matrix inversion, i.e.

$$
\mathbf{F}=(\mathbf{1}-\mathbf{M})^{-1} \mathbf{\Phi} \text {. }
$$

The difficulty with this approach is obviously the size of the matrix: note that the vector $\mathbf{F}$ has dimension $N_{\mathrm{s}}(L+1)^{2}$. However, if the cluster of atoms has some spatial symmetries then $\mathbf{M}$ will assume block form. We demonstrate this with a $2 \times 2$ square cluster of $N_{\mathrm{s}}=4$ atoms perpendicular to the optical axis, scattering in s-waves only. The lengths of the vectors $\boldsymbol{r}_{i}$ from the point source (on

\footnotetext{
${ }^{1}$ All phase shifts used in this paper are courtesy of M.A. Van Hove.
} 
the optical axis) are then equal. Thus, all components of $\mathbf{F}$ and $\boldsymbol{\Phi}$ are equal and $\mathbf{M}$ has the simple structure

$$
\mathbf{M}=\left(\begin{array}{cccc}
0 & M_{1} & M_{1} & M_{2} \\
M_{1} & 0 & M_{2} & M_{1} \\
M_{1} & M_{2} & 0 & M_{1} \\
M_{2} & M_{1} & M_{1} & 0
\end{array}\right)
$$

where $\quad M_{1}=\tau_{0} a^{-1} \exp (\mathrm{i} k a)$ and $M_{2}=\tau_{0}$ $\exp (\mathrm{i} \sqrt{2} k a) /(\sqrt{2} a)$, with $a=\left|\boldsymbol{r}_{i}-\boldsymbol{r}_{j}\right|$, involve first and second neighbour atoms, respectively. The four components of $\mathbf{F}$, all equal, are then given by

$$
F\left(r_{i}\right)=2 \sqrt{\pi}\left(1-2 M_{1}-M_{2}\right)^{-1} r_{i}^{-1} \exp \left(\mathrm{i} k r_{i}\right) .
$$

For an $N \times N$ square array of atoms with electrons scattering in $(L+1)$ partial waves the number of distinct elements in $\mathbf{M}$ is only $N^{2}(L+1)^{2}$ out of a total of $N^{4}(L+1)^{4}$. Storage of all elements of this matrix will obviously be a problem except for very small $N$ and $L$. We have therefore developed an algorithm for the generation of the matrix $\mathbf{M}$ from the knowledge of its distinct elements only. The method we describe below is readily extended to be applicable to any two- or three-dimensional cluster of atoms with some spatial symmetry, be it a crystalline structure or spiral symmetry in a biological molecule. As a demonstration of the method we look at a planar square cluster, perpendicular to the optical axis, of $N \times N$ atoms that scatter in s-waves only. We enumerate these atoms from 1 to $N$ starting in the upper left corner and proceeding by rows. These are then the labels of the matrix M. The elements in its first row can be characterized by the distances between the first atom and all others. Thus we have

$$
M\left(\boldsymbol{r}_{1}, \boldsymbol{r}_{j}\right)=\tau_{0} \exp \left(\mathrm{i} k a_{1 j}\right) / a_{1 j}
$$

where

$$
a_{1 j}=a\left[\left(j_{0}-1\right)^{2}+N^{-2}\left(j-j_{0}\right)^{2}\right]^{1 / 2}
$$

with $a$ the lattice constant, $j=j_{0}+n N$ for $n=0,1, \ldots, N-1$ and $j_{0}=1,2, \ldots, N$. We note that in the present case $\mathbf{M}$ is symmetric with zeroes along the main diagonal. To obtain the other elements divide the matrix $\mathbf{M}$ into $N^{2}$ submatrices of size $N \times N$. Each of these is also symmetric with respect to reflection along the main and also the second diagonal. For the element $M_{i j}=M_{j i}$, we assume $i<j$ and decompose them as

$$
i=n N+i^{\prime}, \quad i^{\prime}=1, \ldots, N, \quad n=0, \ldots, N-1,
$$

$j=m N+j^{\prime}, \quad j^{\prime}=1, \ldots, N, \quad m=0, \ldots, N-1$.

We then find that for $i^{\prime}<j^{\prime}$ and $n<m$ :

$$
\begin{aligned}
M_{n N+i^{\prime}, m N+j^{\prime}} & =M_{(n-1) N+i^{\prime},(m-1) N+j^{\prime}}=\ldots \\
& =M_{i^{\prime},(m-n) N+j^{\prime}}=M_{j^{\prime},(m-n) N+i^{\prime}} \\
& =\cdots=M_{1,(m-n) N+j^{\prime}-i^{\prime}+1} .
\end{aligned}
$$

For more complicated clusters, e.g. three-dimensional clusters or clusters in which scattering includes higher partial waves, this method can be generalized accordingly.

After having solved the problem of storing (or rather calculating) the elements of the matrix $\mathbf{M}$ we can now address the problem of solving Eq. (9). A straightforward iteration procedure often produces sufficient convergence which can sometimes be improved by using the Gauss - Seidel method or similar algorithms.

\section{Simulation of images}

From the wave function (1) one calculates the image on a screen a distance $L$ away as

$$
\hat{I}(\boldsymbol{r})=\frac{L}{r}\left|\psi_{\text {out }}(\boldsymbol{r})\right|^{2}=\frac{L}{r^{3}}|1+\tilde{\psi}(\boldsymbol{r})|^{2},
$$

where we have assumed a spherical incoming wave for simplicity. The factor $L / r$ is required because the image intensity is given by the flux of electrons arriving at the screen and not by their probability density. One usually subtracts the background flux from Eq. (17) to get

$$
I(\boldsymbol{r}) \equiv \hat{I}(\boldsymbol{r})-\frac{L}{r^{3}}=\frac{L}{r^{3}}\left[2 \operatorname{Re}(\tilde{\psi}(\boldsymbol{r}))+|\tilde{\psi}(\boldsymbol{r})|^{2}\right] .
$$




\section{Reconstruction}

At the time of proposing holography with point sources Gabor also suggested a scheme to reconstruct the three-dimensional object wavefront from a two-dimensional hologram. It is based on the Kirchhoff-Helmholtz transform,

$$
K(\boldsymbol{r})=\frac{1}{4 \pi} \int \mathrm{d}^{2} \xi I(\boldsymbol{\xi}) \exp (\mathrm{i} k \boldsymbol{\xi} \cdot \boldsymbol{r} / \xi),
$$

where the integration extends over the 2-dimensional surface of the screen with coordinates $\boldsymbol{\xi}=(X, Y, L)$, a distance $L$ from the source. It has been shown previously that this formula works very well for simulated images calculated for the LEEPS microscope and also for the reconstruction of experimental images, both for electron as well as for optical in-line holography [15].

\section{Examples}

We now turn to examples to examine the role of multiple scattering on image formation and on the quality of reconstruction. In Fig. 1, we show the simulated LEEPS images originating from a
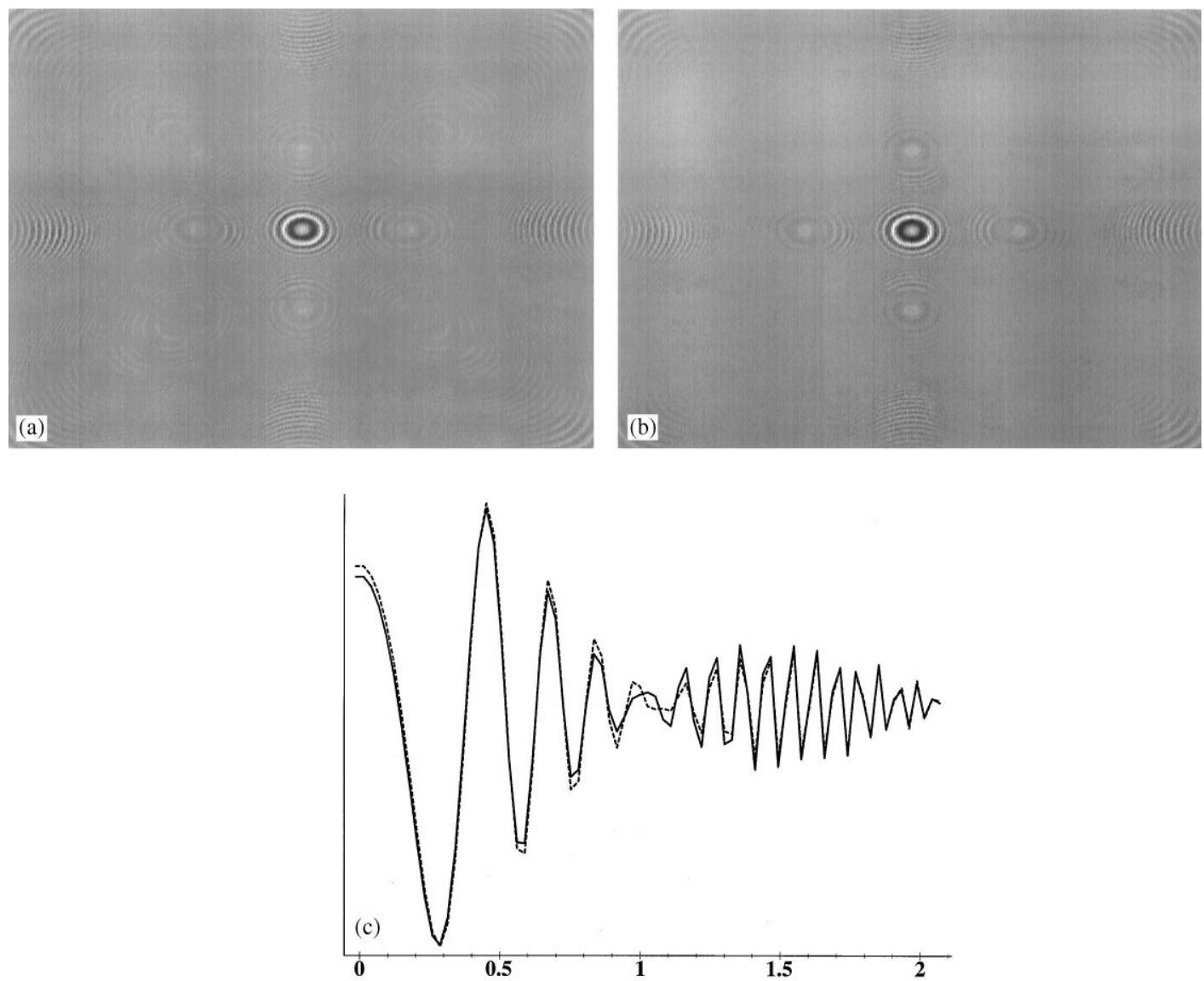

Fig. 1. Simulated LEEPS images for a carbon BCC crystal of three layers with $5 \times 5,4 \times 4$ and $5 \times 5$ atoms, respectively, with a lattice constant of $2.5 \AA$, and with a separation between layers of $4.5 \AA$. The electron energy is $95 \mathrm{eV}$; the distance from source to object is $1000 \AA$, and the source to screen distance is $10 \mathrm{~cm}$. The screen size is $14 \times 14 \mathrm{~cm}^{2}$. The image in part (a) is calculated in the single scattering approximation, and the image in part (b) is for multiple scattering. In both cases, phase shifts up to five partial waves have been included in the calculation. Part (c) compares the intensities along the diagonals of the holograms, with solid and dashed lines for multiple and single scattering, respectively. 
carbon cluster consisting of three layers of $5 \times 5$, $4 \times 4$, and $5 \times 5$ atoms each, arranged on a BCC lattice, calculated in the single scattering approximation and also taking full account of multiple scattering. The distance $a$ between neighbouring atoms within each layer is $a=2.5 \AA$, while the separation $d$ between layers is $d=4.5 \AA$. The electron energy was $95 \mathrm{eV}$ and phase shifts in five partial waves have been included in the calculation. The differences arising from the use of the single scattering approximation as compared with multiple scattering can best be visualized by comparing the intensities in the two holograms along selected cuts, as follows. Two segments from the centre of the image and half way along its diagonal are shown in the Fig. 1(c). Note that both the amplitude and the phase are affected by the single scattering approximation. Although these differences are rather small, they nevertheless are of consequence in the reconstruction, as seen in Fig. 2. We compare in Fig. 2 the reconstructions from the images calculated in the single scattering approximation and for multiple scattering. In Fig. 2(a), the reconstructions are along the optical axis through the central atoms in the two $5 \times 5$ layers, and in Fig. 2(b) the cut is along the diagonal of the front $5 \times 5$ layer. It is clear that at this energy and for this cluster there is hardly any difference in the lateral reconstruction that results from using the single scattering approximation. The difference between the single and multiple scattering reconstructions in depth is noticeable, even though both clearly reveal the two atomic layers.

Can we conclude from this example that multiple scattering is always unimportant, in the sense that it will not ruin the reconstruction? In order to address this question, we present several examples where cluster size and shape is examined, the electron energy is varied, and where the strength of atomic scattering is varied.

We next present results for atomic clusters exhibiting differences in size and shape. We will consider cases where the cluster has several atoms in layers lateral to the optical axis, but is "thin" in the optical direction, i.e. there are relatively few layers in the cluster. We compare this to the opposite extreme, namely where the cluster has several layers of atoms in the optical direction and

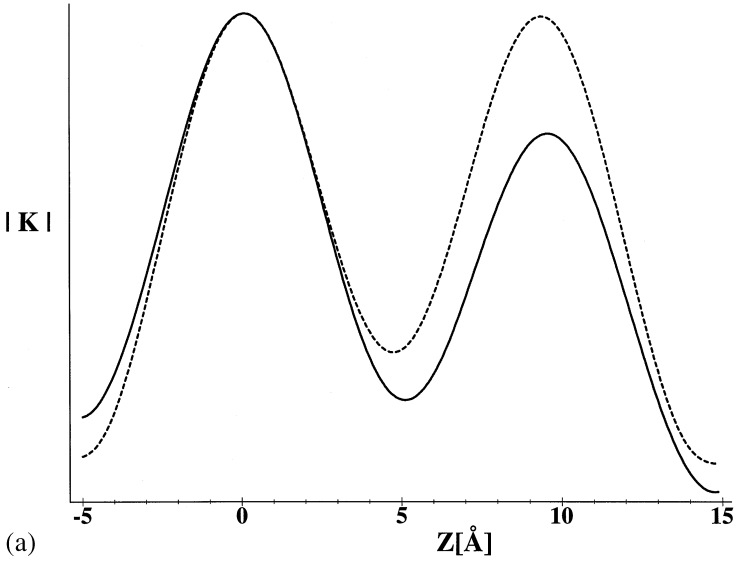

(a)

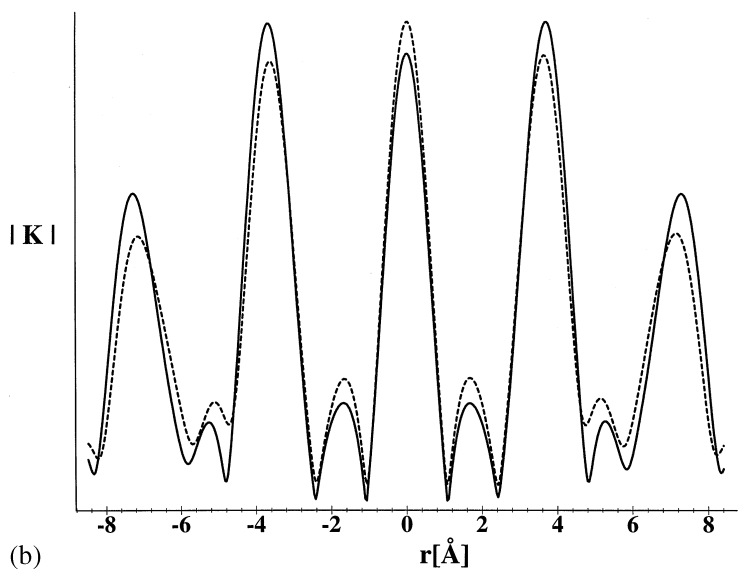

Fig. 2. Reconstruction of images in Fig. 1. The cut in (a) is along the optical axis through the central atoms of the $5 \times 5$ layers; in (b) the cut is along the diagonal of the first $5 \times 5$ layer; $|K|$, given by Eq. (19), is plotted vs. distance $z$ along the optical axis in (a), and vs. distance $r$ along the diagonal in (b). The first layer in the cluster is located at $z=0$, while $r=0$ gives the location of the central atoms in the $5 \times 5$ layers. The solid lines are for multiple scattering; the dashed lines are for single scattering.

has relatively few atoms in layers lateral to the optical axis in each layer. An example of the former is the cluster described above, which we denote by $5|4| 5$. We will refer to this cluster as a "thin" cluster. An example of a cluster thick in the optical direction is a $5|4| 5|4| 5|4| 5|4| 5|4| 5$ cluster. We will refer to such a cluster as a "thick" cluster.

The cuts across the diagonal of the first $5 \times 5$ layer, and along the optical axis, in Figs. 2(a) and (b), show that, even though there is a relatively 
large number of atoms (66) in the cluster, the reconstructions for both single and multiple scattering clearly reveal atomic resolution.

For clusters that are thicker in the optical direction, the reconstructions for single and multiple scattering can be noticeably different. In Fig. 3(a) we show reconstructions for a cut along the optical axis for the $5|4| 5|4| 5|4| 5|4| 5|4| 5$ cluster. Both single and multiple scattering pick out the 6 central atoms in the $5 \times 5$ layers. The plot shown is for $L=4$; with increasing $L$, one expects the multiple scattering reconstruction to get worse.

| K |

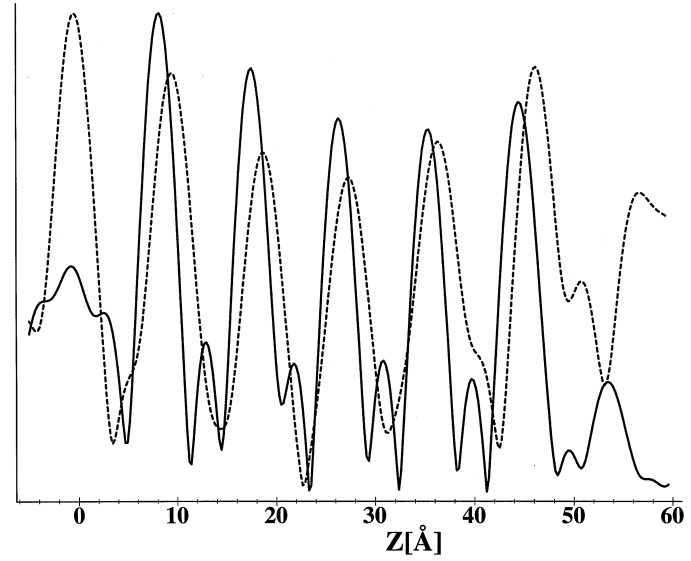

(a)

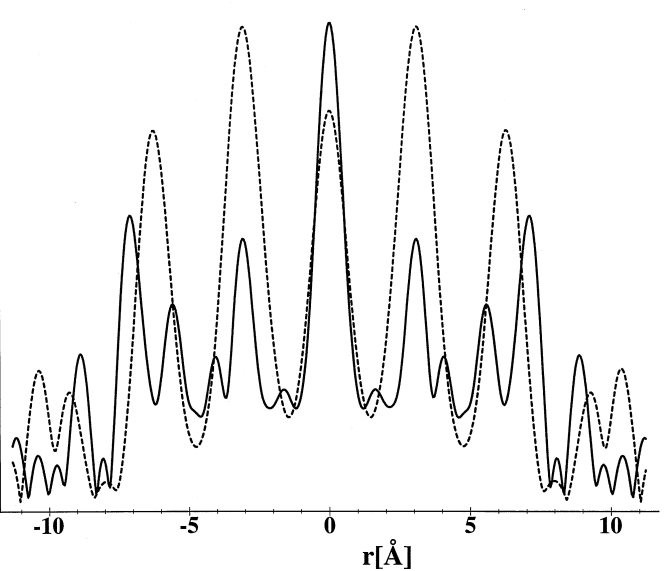

(b)

Fig. 3. Reconstructions for a $5|4| 5|4| 5|4| 5|4| 5|4| 5$ carbon cluster. The cuts are (a) along the optical axis, through the central atoms of the $5 \times 5$ layers, (b) diagonally across the third $5 \times 5$ layer. Single scattering (dashed lines) clearly shows atomic resolution both laterally and in depth. Multiple scattering (solid lines) also reveals the atomic structure, albeit not quite as clearly. In these plots, the electron energy is $E=150 \mathrm{eV}$, and $L=4$.
That multiple scattering still reveals the atomic positions is not restricted to the optical direction, as is clear from Fig. 3(b), which shows a diagonal cut across the third $5 \times 5$ layer. Single scattering again clearly indicates 5 atoms, as does the reconstruction for multiple scattering, although not quite as clearly.

The principal difference between the cases of Figs. 2 and 3 is the thickness of the cluster in the optical direction.

In Figs. 4 and 5, we show reconstructions for gold, a stronger scatterer than carbon. Compared

(a)
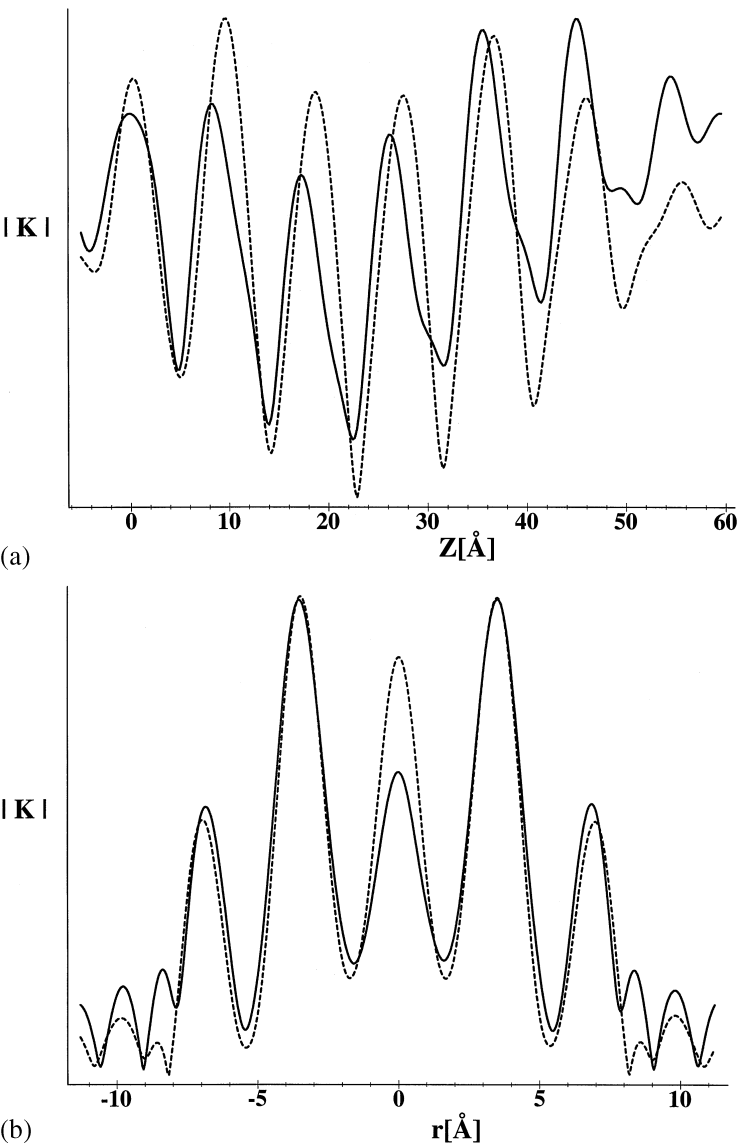

Fig. 4. Reconstructions for a $5|4| 5|4| 5|4| 5|4| 5|4| 5$ gold cluster. The cuts are (a) along the optical axis, through the central atoms of the $5 \times 5$ layers, (b) diagonally across the third $5 \times 5$ layer. Again, both single scattering (dashed lines) and multiple scattering (solid lines) show atomic resolution both laterally and in depth, although the clarity is slightly better for single scattering. In these plots, the electron energy is $E=95 \mathrm{eV}$, and $L=4$. 


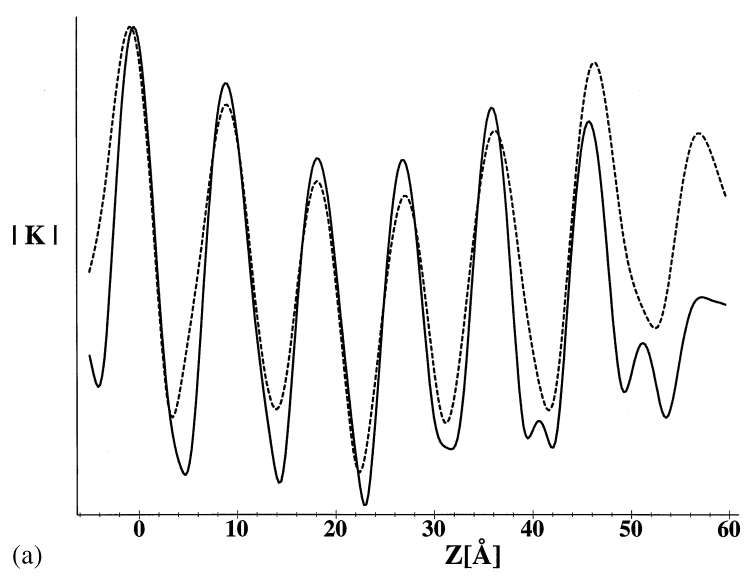

(a)

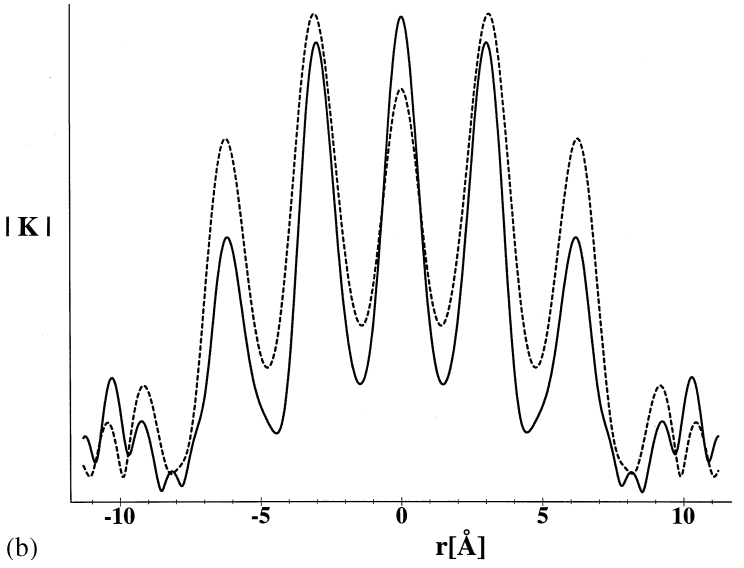

Fig. 5. As in Fig. 5 but for $E=150 \mathrm{eV}$.

with the figures for carbon clusters, we see clearly that, even though we have stronger scattering, the reconstructions still manage to reveal much of the true atomic structure. This is true both in depth and lateral to the optical axis.

We also point out that, in Figs. 3(a) and 4(a), the locations of the peaks for multiple and single scattering are slightly different and that the multiple scattering peaks are actually located noticeably closer to the positions of the atoms than are the single scattering peaks. For completeness, we note that in some of the figures there are some "spurious peaks", i.e. peaks in the reconstructions that occur at positions where there are no atoms. Such spurious peaks are by no means unexpected (see, e.g. Ref. [13]). Methods for eliminating such spurious peaks have been investigated in previous works (see below).
At this point, it is important that we address the issue of convergence of the series in Eq. (6). We have examined the convergence properties of this equation for a large number of examples. In general, we find that the series converges, and to the correct solution (see below) for "thick" clusters only if $L=0$. The reason we cannot be satisfied with just the $L=0$ results is clearly conveyed in Fig. 6, where we show reconstructions for various carbon clusters with $L=0$. The clusters are: (a) a (3|2)-type cluster, with $203 \times 3$ layers; (b) a (5|4)-type cluster, with $205 \times 5$ layers; and (c) a (9|8)-type cluster, with $209 \times 9$ layers. In all three cases, multiple and single scattering show very similar reconstructions along the optical axis. The same is true for lateral reconstructions. Given that the exact solutions for $L=0$ give negligible difference between multiple and single scattering, while the exact solution for $L=5$ for the $5|4| 5$ cluster gives a distinct difference along the optical axis [as is evident from Fig. 2(b)], we have no choice but to examine $L>0$. We must therefore obtain the best possible results for $L>0$, even if they are not exact results. In doing so, we find that, for thick clusters, the series approach limiting values as successive terms are added in; i.e. the series for a given cluster and a given value of $\mathrm{L}$ will approach a limiting, or "best" value, provided we sum an "optimal" number of terms. Proceeding beyond this optimal number results in a departure from the limiting value, with the sum diverging as more and more terms are added. This type of limited summation is by no means particular to the problem studied in this paper, and is indeed often seen in mathematical situations; a good example is the case of series often referred to as "asymptotic series". For such series, one can obtain approximate, but not arbitrarily accurate, solutions to the problem at hand. The best approximation is obtained by summing an optimal number of terms, just as in the case investigated here. That is, the series in Eq. (6) exhibits behaviour like that of these asymptotic series. As such, in order to capture the essential physics, we must content ourselves with the best solution possible, and terminate the power series at the optimal point. We emphasize that the results shown in Figs. 3-5 are the best that can be obtained, and these results 
have the following limitation. Although the series "converges" for an optimal number of terms, the ratio of a given element in $\boldsymbol{\Phi}_{\mathbf{F}} \equiv(\mathbf{1}-\mathbf{M}) \mathbf{F}$, to the corresponding element in $\boldsymbol{\Phi}$ is not necessarily close to unity, even though $\mathbf{F}$ is the best solution one can obtain (recall Eq. (9): $(\mathbf{1}-\mathbf{M}) \mathbf{F}=\boldsymbol{\Phi})$. The consequence of this is that further work, beyond the scope of this paper, may be needed to establish definitively where multiple and single scattering present different pictures of the atomic structure of the cluster. For completeness, we also point out that the cluster size and $L$-values we have used are the largest that can be examined within our computational limitations.

Evidence supporting our view that the results presented in this work convey the correct physical

(a)

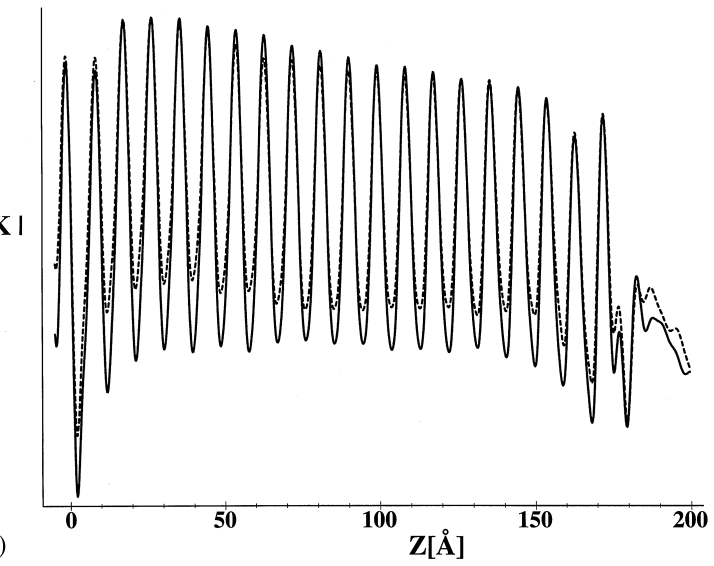

behaviour is given in Fig. 7, where we present exact reconstructions for a (3|2)-type cluster with $203 \times 3$ layers, with $E=122 \mathrm{eV}$ and $L=4$. Fortuitously, for this cluster and this electron energy, the convergence of the series is exact $\left(\boldsymbol{\Phi}_{\mathbf{F}} \equiv(\mathbf{1}-\mathbf{M}) \mathbf{F}=\boldsymbol{\Phi}\right)$. That atomic resolution is obtained along the optical axis in this case, even for multiple scattering including partial waves up to $L=4$, strongly supports the claim that the correct physical behaviour has been captured by the procedure we have chosen to employ.

Further evidence that there is good reason to believe that the figures in this paper do indeed convey the actual physical features of the clusters studied for multiple scattering, we present Fig. 8. Fig. 8 shows reconstructions along the optical axis,

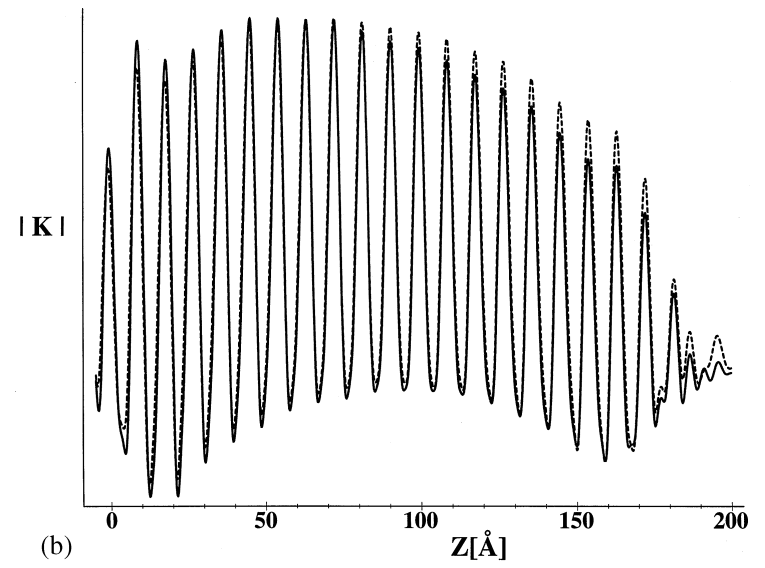

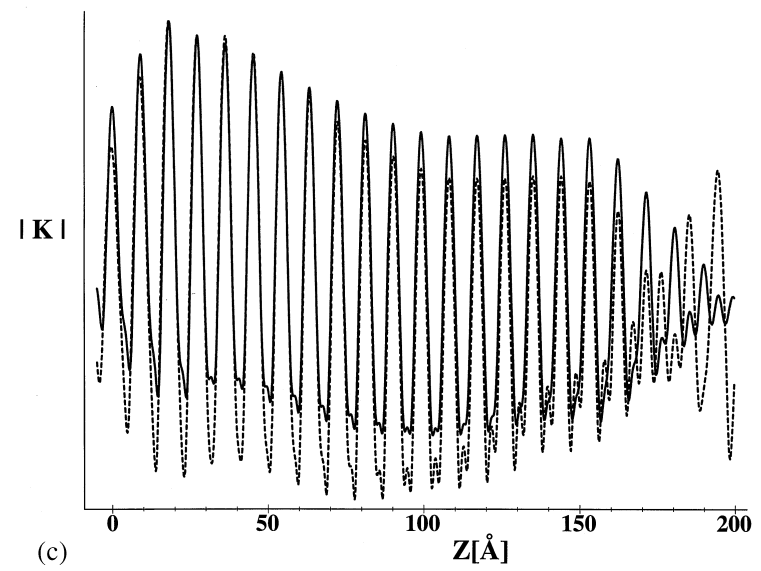

Fig. 6. Reconstructions for various carbon clusters with $L=0$. The clusters are: (a) a (3|2) -type cluster, with $203 \times 3$ layers; (b) a $(5 \mid 4)$-type cluster, with $205 \times 5$ layers; and (c) a (9|8)-type cluster, with $209 \times 9$ layers. In all cases, the cuts are along the optical axis through the geometrical center of the cluster, and the electron energy is $E=95 \mathrm{eV}$. 


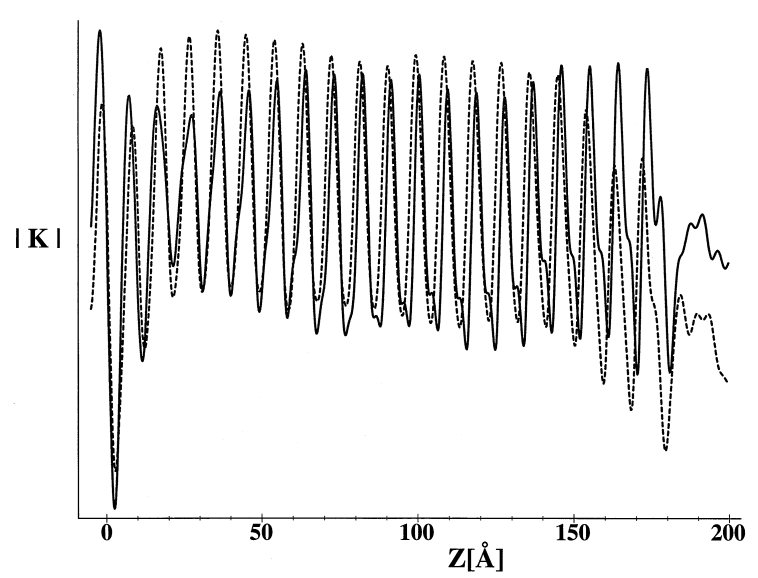

Fig. 7. Reconstructions for a (3|2)-type carbon cluster, with $203 \times 3$ layers; $L=4$ and $E=122 \mathrm{eV}$. The cut is along the optical axis through the geometrical centre of the cluster. For this cluster and this electron energy, even with $L>0$, the convergence is exact. See text for full discussion.

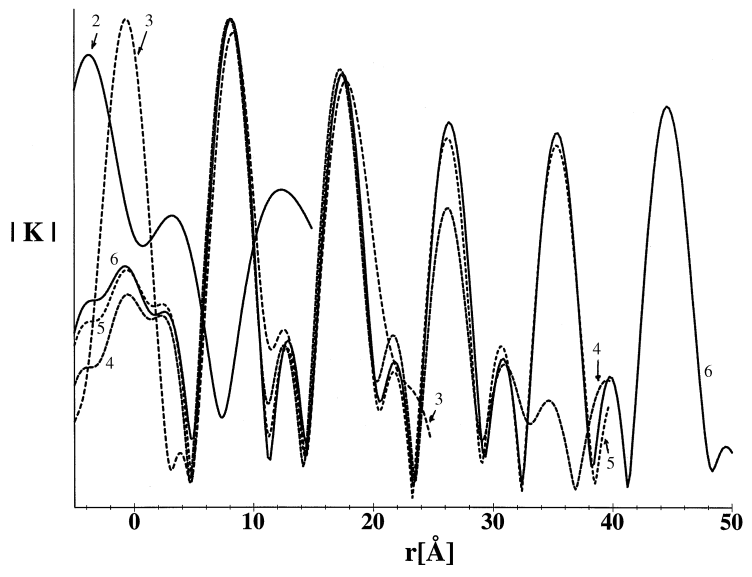

Fig. 8. Reconstructions along the optical axis, through the central atoms of the $5 \times 5$ layers, of a sequence of $(5 \mid 4)-$ type carbon clusters. Each curve is labeled by the number of $5 \times 5$ layers in the cluster. $L=4$ and $E=150 \mathrm{eV}$ for each cluster. The curves reveal a systematic trend, which supports the idea that these curves, while not exact solutions, are nevertheless conveying the principal physical effects involved. See text for further discussion.

through the central atoms of the $5 \times 5$ layers, of a sequence of $(5 \mid 4)$-type carbon clusters. In the figure are curves for $5|4| 5,5|4| 5|4| 5,5|4| 5|4| 5|4| 5$, $5|4| 5|4| 5|4| 5|4| 5$, and $5|4| 5|4| 5|4| 5|4| 5|4| 5$ clusters. We note that these curves reveal a systematic trend. Each of the curves was obtained by finding the best convergence for $L=4$ for each cluster.
That there is a systematic trend in the curves, and no indication of swings in the behaviour from one cluster to the next, supports the idea that, even though exact solutions cannot be obtained, these "best" solutions are nevertheless conveying the principal physical effects involved.

There are of course cases where there is no convergence at all. In such cases, one cannot obtain any meaningful reconstruction from Eq. (6). It would seem that such cases correspond to multiple scattering in higher partial waves as being too strong. The results we have given are for situations where the multiple scattering, although important, still gives meaningful reconstructions.

We note that the physical reason for the sensitivity in the power series is that not all of the physical processes involved in multiple scattering are captured in the power series. The terms that have been discarded are complicated enough to render their inclusion as impractical. Thus, an alternative to the manner in which we have decided to address this situation is to include other terms in the multiple scattering, an alternative which necessitates an enormous amount of work. We thus have no choice but to forego this alternative route and are thus left with the series in Eq. (6).

As a result of this issue of convergence, we have examined a large number of cases, varying the geometry of the cluster and the following length scales: the interatomic spacing within each layer, $a$, the spacing between successive layers, $d$, and the electron wavelength, $\lambda$ (i.e. the electron energy $E$ ). We have studied many examples, enough to be convinced that the principal physical effects have been captured by our approach. The results we have presented in this paper should be viewed with this issue of convergence in mind.

\section{Multiple scattering in LEED vs. LEEPS}

Multiple scattering is also important in the study of low-energy electron diffraction (LEED). We next compare multiple scattering in LEED with our work on multiple scattering in LEEPS.

The methods used in the interpretation of pointsource electron-scattering hologram intensities in LEEPS microscopy are very similar to those 
employed in LEED theory. The main differences between the two are the methods of reconstruction of the holograms, and the distances between the source of the electron waves and the atomic sites under study. In LEEPS microscopy, the pointsource electron waves are emitted at large distances from the object, relative to the atomic distances within the object, while in the holographies employed in LEED, the electrons are emitted within atomic distances from the object. The holographies in LEED are primarily distinguished from one another by their source of electrons, e.g. photoelectron diffraction, Auger decay, etc. The primary difference in the equations governing the holographies of LEED and LEEPS is that, in LEEPS the point-source of spherical electron waves is at a large distance from the object. This allows considerable simplification in the analytic equations [13]; multiple scattering in LEED is even more complicated than in LEEPS.

With this in mind, the discussion of our results is incomplete without a comparison of our results to those previously obtained in LEED. We begin with a discussion of the methods used to account for multiple scattering and then consider the results that have been observed in LEED calculations. The methods used to take account of multiple scattering in LEED applications can be classified into two groups: cluster methods and perturbative methods. The perturbative method $[16,17]$, referred to as a "path" approach, takes the analytical expressions for the amplitude of a scattered electron wave and derives a new, separable, representation for the Green's function matrix. This representation allows approximations that result in computationally efficient equations. In this approach, it is implicitly assumed that the multiple scattering expansion will converge. As such, it is necessary to complement these calculations with exact calculations which can be time consuming unless convergence is a priori known. Further, in order to evaluate the intensity of scattered electron waves, it is necessary to determine the scattering paths to be used, and a sufficiently large number of scattering paths must be included to attain accurate results. This is a much faster approach than a full matrix inversion. In our algorithm, we exploit the symmetry of the structure matrix, and use a series representation for the inverse, which, when convergent, converges rapidly, and is orders of magnitude faster than a full numerical matrix inversion. Moreover, our method for computing the hologram taking full account of multiple scattering is equivalent to summing the path formalism to infinite order, and is therefore very accurate.

Work has been done to improve the efficiency of the cluster method [18-20] for calculating the diffraction patterns in LEED. In these works, the analytical expressions are cast in a form such that the numerical evaluation of multiple scattering terms can be done in an efficient manner. For example, by appropriate rotations of the coordinate system, and expanding the electron wave about a new origin (the center of a scattering potential), Fritzshe [18] expresses the coefficients of the free electron propagator in an angular momentum representation in an alternate form, deriving computationally efficient recurrence relations for the appropriate quantities involved. This significantly improves the speed of the calculations. Saldin et al. find computationally efficient equations by considering the atoms surrounding an emitter atom in concentric shells, allowing the classification of processes into "intershell" and "intrashell" processes, and employing the separable Green's functions of Rehr [17] along with a Taylor series magnetic quantum number expansion and a reduced angular momentum expansion. The equations become much more computationally feasible in these cases. It is nevertheless still necessary to include multiple scattering term-byterm, i.e. single scattering, then double scattering, and so on. In our method, we account for all possible multiple scattering simultaneously. It is also worth noting that in the cases where only nearest-neighbor scattering is important, our algorithm is extremely efficient in the sense that the structure matrix retains its high degree of symmetry while it also becomes a sparse matrix. The computations outlined in this paper, when dealing with a sparse structure matrix having a high degree of symmetry, are extremely efficient for including multiple scattering terms.

We have seen in this paper that multiple scattering does not significantly degrade the 
quality of the images obtained. This has also been seen in LEED theory [20] where the reconstructed images of an Auger emission reconstruction of an $\mathrm{O}$ atom on a $\mathrm{Ni}\left(\begin{array}{lll}1 & 0 & 0\end{array}\right)$ surface gave results for single and multiple scattering that were very similar. Saldin et al. did show, however, that there were very noticeable differences in the calculated diffraction patterns, in contrast to our findings that the holograms for single and multiple scattering are very similar. This can be attributed to the geometry of the emission of the probing electron wave. As mentioned previously, in Auger emission applications, the electron source is within atomic distances of the object, while in LEEPS microscopy the electron source is at a large distance from the atomic cluster. It has also been shown that multiple scattering does tend to obscure images in some LEED applications, and that improvements can be made in reconstructing the holograms [21,22]. Images in some LEED applications are also obscured by "twin" images, which are virtually not present in LEEPS images due to the large distances from source to object. It was shown that by energy averaging [21], and averaging over the angle of collection [22] the effects of multiple scattering on reconstructed images could be significantly reduced. That is, the quality of the images were improved by averaging holograms of different energies and screen positions, respectively. This was also shown to work in LEEPS [23,24].

\section{Outlook}

In this paper we have reviewed the theory to calculate electron holograms for the point source electron microscope including multiple scattering. Holographic diffraction and single scattering are dominant for small carbon clusters. We have given examples which show that, even when multiple scattering is important, one can still obtain reconstructions that reveal the atomic structure both along and lateral to the optical axis. This is encouraging in the sense that we can be confident in using the single scattering approximation in many cases of physical interest. Our result that good reconstructions can still be obtained even when multiple scattering is important, has significant implications for experimental work in LEEPS, i.e. in cases where multiple scattering will be appreciable. We have also found, however, that in some cases the multiple scattering is too strong and reconstruction is not possible.

Multiple scattering will become appreciable for large enough clusters, especially for clusters that are thick enough in the optical direction. Multiple scattering will also be appreciable in stronger scatterers, such as metallic films, for which classical diffraction also starts to play a role, and eventually becomes dominant for films of more than just a few atomic layers.

As shown in previous investigations [23,24], one expects that the multiple scattering reconstructions can be improved by taking images at various electron energies and/or at different tilt angles of the screen. Alternatively, by combining a few holograms in the manner described in Ref. [25], improved reconstructions would be obtained for multiple scattering.

The consequence of the issue of convergence described above is that further work is required in order to definitively establish the effect of multiple scattering. We regard the work in this paper as a first step.

\section{Acknowledgements}

This work was supported financially by the Natural Sciences and Engineering Research Council of Canada. We thank Prof. H.J. Kreuzer for constructive comments. We thank the UNBC Mathematics and Computer Science Department for access to their computational facilities. Assistance from Wilson Yeung with various aspects of the computer programs is gratefully acknowledged. We also thank the referee for constructive comments.

\section{References}

[1] D. Gabor, Nature (London) 161 (1948) 777.

[2] H. Lichte, Ultramicroscopy 20 (1986) 293.

[3] A. Tonomura, Rev. Mod. Phys. 59 (1987) 639. 
[4] J.J. Barton, Phys. Rev. Lett. 61 (1988) 1356.

[5] H.-W. Fink, IBM J. Res. Dev. 30 (1986) 460-463.

[6] H.-W. Fink, Phys. Scr. 38 (1988) 260.

[7] W. Stocker, H.-W. Fink, R. Morin, Ultramicroscopy 31 (1989) 379.

[8] H.-W. Fink, W. Stocker, H. Schmid, Phys. Rev. Lett. 65 (1990) 120.

[9] H.-W. Fink, H. Schmid, H.J. Kreuzer, A. Wierzbicki, Phys. Rev. Lett. 67 (1991) 1543.

[10] A. Tonomura, L.F. Allard, D.C. Pozzi, D.C., Joy, Y.A. Ono, (Eds.), Electron Holography, Elsevier Science B.V., Amsterdam, 1995.

[11] A. Degiovanni, R. Morin, 1994, in: Proceedings of ICEM 13, Electron Microscopy 1994, Vol. 1, Les Editions de Physique, Paris.

[12] S. Horch, R. Morin, J. Appl. Phys. 74 (1993) 3652.

[13] H.J. Kreuzer, K. Nakamura, A. Wierzbicki, H.-W. Fink, H. Schmid, Ultramicroscopy 45 (1992) 381.

[14] H.J. Kreuzer, A. Wierzbicki, M.G.A. Crawford, C.B. Roald, in: V.T. Binh, N. Garcia, K. Dransfeld (Eds.), Nanosources and Manipulation of Atoms under High
Fields and Temperatures: Applications, Kluwer Academic Publishers, Dordrecht, 1993.

[15] H. Schmid, H.-W. Fink, H.J. Kreuzer, J. Vac. Sci. Technol. B 13 (1995) 2428.

[16] D. Friedman, C.S. Fadley, J. Electron Spectrosc. Relat. Phenom. 51 (1990) 689.

[17] J.J. Rehr, R.C. Albers, Phys. Rev. B 41 (1990) 8139.

[18] V. Fritsche, J. Phys. Condens. Matter 2 (1990) 1413.

[19] V. Fritsche, J. Phys. Condens. Matter 2 (1990) 9735.

[20] D.K. Saldin, G.R. Harp, X. Chen, Phys. Rev. B 48 (1993) 8234.

[21] J.J. Barton, Phys. Rev. Lett. 67 (1991) 3106.

[22] S.Y. Tong, H. Huang, C.M. Wei, Phys. Rev. B 46 (1992) 2452.

[23] M.R.A. Shegelski, S. Faltus, T. Clark, H.J. Kreuzer, Ultramicroscopy 74 (1998) 169.

[24] M.R.A. Shegelski, M. Reid, T. Clark, S. Faltus, Ultramicroscopy 77 (1999) 129.

[25] M.R.A. Shegelski, T.A. Rothwell, Ultramicroscopy, submitted for publication. 